\title{
Posterior callosotomy using a parietooccipital interhemispheric approach in the semi-prone park-bench position
}

\author{
Hirotaka Ito, MD, Michiharu Morino, MD, PhD, Manabu Niimura, MD, PhD, \\ Sachiko Takamizawa, MD, and Yoshie Shimizu, MD \\ Department of Neurosurgery, Tokyo Metropolitan Neurological Hospital, Tokyo, Japan
}

\begin{abstract}
A 2-stage corpus callosotomy is accepted as a palliative procedure for patients older than 16 years with, in particular, medically intractable generalized epilepsy and drop attack seizures and is preferable for a lower risk of disconnection syndrome. Although the methods by which a previously performed craniotomy can be reopened for posterior callosotomy have already been reported, posterior corpus callosotomy using a parietooccipital interhemispheric approach with the patient in a semi-prone park-bench position has not been described in the literature. Here, the authors present a surgical technique for posterior callosotomy using a parietooccipital interhemispheric approach with a semi-prone parkbench position as a second surgery. Although this procedure requires an additional skin incision in the parietooccipital region, it makes the 2-stage callosotomy safer and easier to perform because of reduced intracranial adhesion, less bleeding, and an easier approach to the splenium of the corpus callosum.
\end{abstract}

http://thejns.org/doi/abs/10.3171/2014.12.JNS141732

KEY WORDS posterior corpus callosotomy; surgical approach; epilepsy surgery; surgical technique

$\mathrm{C}$ ORPUS callosotomy is accepted as a palliative procedure for patients with, in particular, medically intractable generalized epilepsy and drop attack seizures. Although a 1-stage total corpus callosotomy can be performed in patients younger than 16 years, a 2 -stage corpus callosotomy is preferred for patients older than 16 years given its lower risk for disconnection syndrome. ${ }^{14} \mathrm{At}$ some institutions, posterior corpus callosotomy has been performed when the efficacy of anterior corpus callosotomy was insufficient. ${ }^{1,8-10,13}$ We have performed a second surgery for total callosotomy in cases that showed little or no clinical improvement in the 6-month follow-up after anterior partial callosotomy. The previous skin incision and craniotomy were generally reopened in the second surgery ${ }^{6}$ however, difficulties have been associated with this dissection because of intracranial adhesion, which is often observed following surgical intervention, and because of the long distance to the splenium of the corpus callosum, especially in adult cases. On the other hand, the shorter distance to the splenium in a posterior corpus callosotomy with a parietooccipital interhemispheric approach permits a safer, more precise dissection. Here, we present, as a sec- ond surgery, a technique for posterior callosotomy using a parietooccipital interhemispheric approach with a semiprone park-bench position.

\section{Methods}

In this retrospective study, we searched our institutional database for patients with epilepsy and drop attack seizures who had undergone anterior corpus callosotomy and an additional posterior corpus callosotomy for residual seizures in the period from 1999 to 2013. Patients with follow-up periods shorter than 3 months were excluded from our analysis. Magnetic resonance imaging was performed after surgery to evaluate the complete division.

\section{Surgical Techniques}

Magnetic resonance venography was performed in all cases preoperatively to determine which side was favorable for approach. The nondominant venous side was selected as the approach side; for example, when bridging veins were dominant in the right side, the left side was regarded as the approach side. 
After the induction of general endotracheal anesthesia, the patient was secured in a Mayfield head holder and placed in the semi-prone park-bench position lying on the approach side (Fig. 1a). An inverted-U-shaped skin incision was made in the parietooccipital region, and a rectangular bone flap was elevated to expose the paramedian non-venous-dominant hemisphere as well as the superior sagittal sinus. The craniotomy was roughly centered 4 $\mathrm{cm}$ above the inion, with an anteroposterior dimension of approximately $6 \mathrm{~cm}$ and a mediolateral dimension of approximately $4.5 \mathrm{~cm}$ (Fig. 1b). A C-shaped dural incision was made while taking care to preserve the veins, and the interhemispheric fissure was approached via sharp microdissection along the parietooccipital sulcus as a guide to the splenium (Fig. 1c). After reaching the splenium, the pericallosal cistern was opened to expose the corpus callosum. With the use of bipolar cautery and Optoirrigator suction, the corpus callosum was dissected along the midline anteriorly with the line of dissection extending to the posterior edge of the dissected anterior corpus callosum (Fig. 1d) and posteriorly until the vein of Galen and the internal cerebral veins could be visualized via the arachnoid (Fig. 1e). After the total corpus callosotomy was completed (Fig. 1f), closure was conducted in the usual manner.

\section{Results}

Sixty-five epilepsy patients with drop attack seizures underwent corpus callosotomy (91 operations). Patients were clinically followed up for 3-6 months. Posterior corpus callosotomy was additionally performed in 26 of the 65 cases because of residual drop attack seizures. All operations were performed safely. Magnetic resonance imaging after surgery showed complete division of the corpus callosum in all cases. Complications were observed
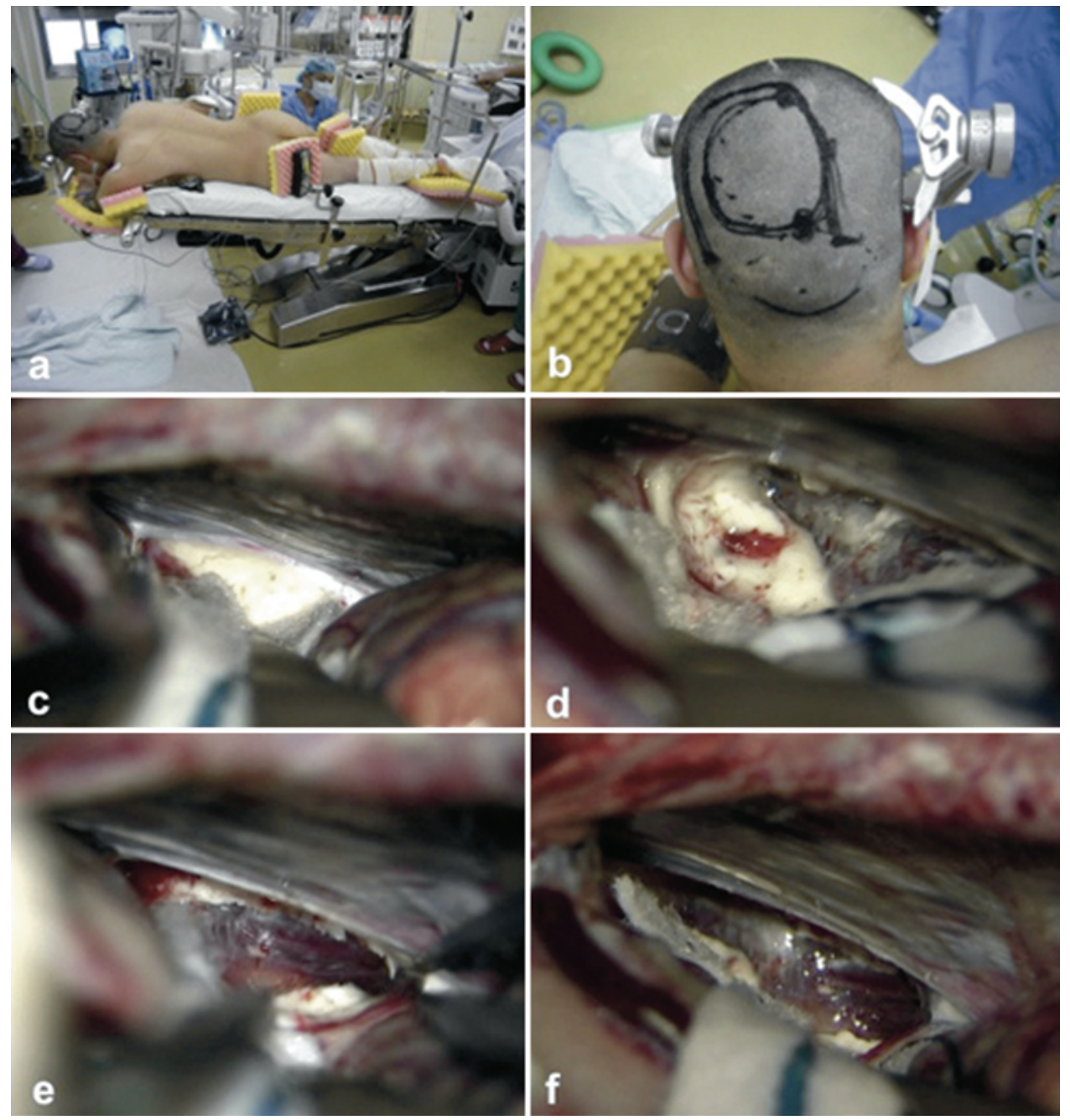

FIG. 1. a: The patient was secured in a Mayfield head holder and was placed in the semi-prone park-bench position lying on the approach side. b: An inverted-U-shaped skin incision was made in the parietooccipital region. Craniotomy was roughly centered $4 \mathrm{~cm}$ above the inion, with an anteroposterior dimension of approximately $6 \mathrm{~cm}$ and a mediolateral dimension of approximately $4.5 \mathrm{~cm}$. c: Approach into the interhemispheric fissure via sharp microdissection along the parietooccipital sulcus as a guide to the splenium. $d$ : The corpus callosum was divided anteriorly with the line of dissection extending to the posterior edge of the dissected anterior part of the corpus callosum. e: After the posterior part of the corpus callosum had been divided, the vein of Galen and internal cerebral veins could be visualized via the arachnoid. f: Completion of a total corpus callosotomy. Figure is available in color online only. 
in 3 of the 26 cases: 2 cases of transient disconnection syndrome including aphasia and left-sided apraxia and 1 case of epidural abscess. Individual patient information, including complications, is summarized in Table 1.

\section{Discussion}

There are 2 palliative surgeries for medically intractable epilepsy and drop attack seizure: corpus callosotomy and vagus nerve stimulation (VNS). It is true that corpus callosotomy is associated with a higher risk for complications and that VNS is less invasive and reversible with fewer serious complications; however, corpus callosotomy is equally or more efficacious than VNS for primarily generalized seizure and drop attack seizure. ${ }^{3,711}$ Complications associated with corpus callosotomy include hydrocephalus, meningitis, and cerebral edema, ${ }^{5}$ although acute disconnection syndrome and split-brain syndrome are the most problematic complications following total callosotomy. Previous studies have suggested that 1-stage total callosotomy should be avoided if patients are older than 10 years of age $\mathrm{e}^{12}$ and that 2-stage corpus callosotomy is preferable for a lower risk of disconnection syndrome. ${ }^{14}$ Although the methods by which a previously performed craniotomy was reopened for posterior callosotomy have been reported, ${ }^{6}$ the posterior corpus callosotomy using a parietooccipital interhemispheric approach with the patient in a semi-prone park-bench position has not been described. The posterior interhemispheric approach in a lateral position has been described as effective for exposing lesions in the posterior midline with reduced bleeding, shorter operative durations, decreased postoperative cerebral edema, and improved visual outcomes. ${ }^{2}$ The benefits of the parietooccipital interhemispheric approach were as follows. Since venous pressure was lower in the semi-prone park-bench position than in the prone position, intraoperative bleeding could be minimized. Positioning the patient lying on the approach side allowed the occipital and parietal lobes to fall aside with the aid of gravity, ${ }^{15}$ and both cerebral hemispheres could be separated with minimum retraction, which reduces the incidence and severity of long-lasting disconnection syndrome. ${ }^{4}$ The craniotomy was centered on the parietooccipital sulcus to reach the splenium in the shortest distance, and the corpus callosum could then be more safely and precisely divided (Fig. 2). Although this procedure poses a cosmetic problem because of the additional skin incision in the parietooccipital region, it is a safe and effective way to perform 2-stage corpus callosotomy without any morbidity such as disconnection syndrome. The advantages and disadvantages associated with this procedure have been outlined in Table 2.

TABLE 1. Individual patient information, including complications*

\begin{tabular}{|c|c|c|c|c|}
\hline Case No. & Age at Op (yrs), Sex & Seizure Etiology & Seizure Type & Complications \\
\hline 1 & $16, \mathrm{M}$ & Unknown & Multiple seizure type w/ drop attack & None \\
\hline 2 & $18, \mathrm{M}$ & Frontal arachnoid cyst & Drop attack & None \\
\hline 3 & $24, M$ & Unknown & Drop attack & None \\
\hline 4 & $23, \mathrm{~F}$ & Unknown & Drop attack, CPS & None \\
\hline 5 & $24, \mathrm{M}$ & Cortical dysplasia & Multiple seizure type w/ drop attack & Transient disconnection syndrome \\
\hline 6 & $42, \mathrm{M}$ & Lennox-Gastaut & Drop attack, tonic seizure & None \\
\hline 7 & $19, \mathrm{M}$ & Unknown & Drop attack, tonic seizure & Transient disconnection syndrome \\
\hline 8 & $26, \mathrm{M}$ & Unknown & Drop attack, tonic seizure & None \\
\hline 9 & $43, \mathrm{~F}$ & Lennox-Gastaut & Drop attack & None \\
\hline 10 & $17, \mathrm{~F}$ & Unknown & Drop attack & None \\
\hline 11 & $14, \mathrm{M}$ & Hypoxic ischemic encephalopathy & Absence, tonic seizure & None \\
\hline 12 & $25, \mathrm{~F}$ & Chromosome aberration ( 15 trisomy) & Drop attack & None \\
\hline 13 & $12, \mathrm{M}$ & Lennox-Gastaut & Drop attack & None \\
\hline 14 & $30, \mathrm{~F}$ & Lennox-Gastaut & Drop attack & None \\
\hline 15 & $26, \mathrm{~F}$ & Unknown & Drop attack & None \\
\hline 16 & $16, \mathrm{M}$ & Progressive myoclonus & Myoclonus, tonic seizure & None \\
\hline 17 & $33, \mathrm{~F}$ & Progressive myoclonus & Myoclonus, tonic seizure & None \\
\hline 18 & $23, \mathrm{M}$ & Acute encephalitis & Drop attack & None \\
\hline 19 & $28, M$ & Hypoxic ischemic encephalopathy & Drop attack & None \\
\hline 20 & $52, \mathrm{M}$ & Lennox-Gastaut & Drop attack & None \\
\hline 21 & $35, \mathrm{~F}$ & Lennox-Gastaut & Absence, tonic seizure & None \\
\hline 22 & $31, \mathrm{M}$ & Unknown & Drop attack, myoclonus, GTCS & Epidural abscess \\
\hline 23 & $21, \mathrm{~F}$ & Unknown & Drop attack, tonic seizure, absence & None \\
\hline 24 & $37, \mathrm{M}$ & Lennox-Gastaut & Drop attack & None \\
\hline 25 & $30, M$ & Lennox-Gastaut & Drop attack & None \\
\hline 26 & $43, \mathrm{M}$ & Lennox-Gastaut & Drop attack & None \\
\hline
\end{tabular}

CPS = complex partial seizure; GTCS = generalized tonic-clonic seizure.

* All patients were right handed. 
TABLE 2. Advantages and disadvantages of each position for posterior callosotomy

\begin{tabular}{lll}
\hline \multicolumn{1}{c}{ Position } & \multicolumn{1}{c}{ Advantages } & Disadvantages \\
\hline Supine & No additional skin incision; less blood loss & $\begin{array}{c}\text { Difficult to dissect because of postoperative adhesion \& } \\
\text { long distance to ampulla of corpus callosum }\end{array}$ \\
\hline Prone & $\begin{array}{c}\text { Easy to dissect because of short distance to ampulla } \\
\text { of corpus callosum \& less adhesion }\end{array}$ & $\begin{array}{c}\text { Additional skin incision; more blood loss because of } \\
\text { higher venous pressure }\end{array}$ \\
\hline $\begin{array}{c}\text { Semi-prone } \\
\text { park-bench }\end{array}$ & $\begin{array}{c}\text { Easy to dissect because of short distance to ampulla } \\
\text { of corpus callosum \& less adhesion; less blood } \\
\text { loss }\end{array}$ & \begin{tabular}{c} 
Additional skin incision \\
\hline
\end{tabular} \\
\hline
\end{tabular}

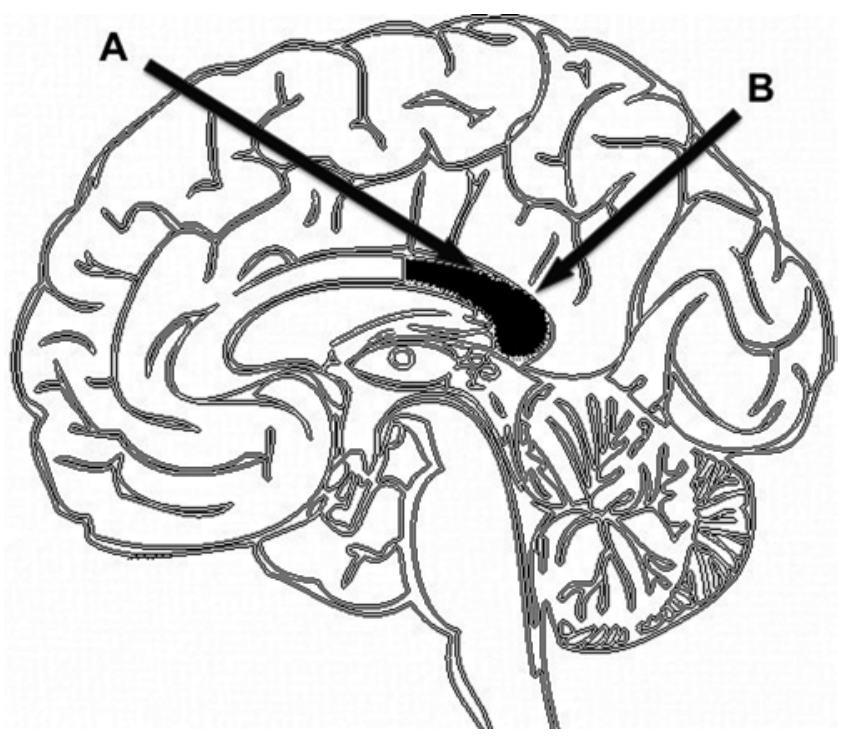

FIG. 2. Drawing of the surgical approach. $A$ and $B$ show the approaches to the splenium of the corpus callosum from the frontal region and the parietooccipital region, respectively. The distance to the splenium of the corpus callosum is shorter in B than in A. Copyright Michiharu Morino. Published with permission.

\section{Conclusions}

Posterior callosotomy using a parietooccipital interhemispheric approach with the patient in the semi-prone park-bench position makes 2-stage callosotomy safer and easier to perform.

\section{References}

1. Cendes F, Ragazzo PC, da Costa V, Martins LF: Corpus callosotomy in treatment of medically resistant epilepsy: preliminary results in a pediatric population. Epilepsia 34:910-917, 1993

2. Chi JH, Lawton MT: Posterior interhemispheric approach: surgical technique, application to vascular lesions, and benefits of gravity retraction. Neurosurgery 59 (1 Suppl 1):ONS41-ONS49, 2006

3. Cukiert A, Cukiert CM, Burattini JA, Lima AM, Forster CR, Baise C, et al: Long-term outcome after callosotomy or vagus nerve stimulation in consecutive prospective cohorts of children with Lennox-Gastaut or Lennox-like syndrome and non-specific MRI findings. Seizure 22:396-400, 2013

4. Fuiks KS, Wyler AR, Hermann BP, Somes G: Seizure out- come from anterior and complete corpus callosotomy. J Neurosurg 74:573-578, 1991

5. Jalilian L, Limbrick DD, Steger-May K, Johnston J, Powers AK, Smyth MD: Complete versus anterior two-thirds corpus callosotomy in children: analysis of outcome. J Neurosurg Pediatr 6:257-266, 2010

6. Kim DS, Yang KH, Kim TG, Chang JH, Chang JW, Choi JU, et al: The surgical effect of callosotomy in the treatment of intractable seizure. Yonsei Med J 45:233-240, 2004

7. Lancman G, Virk M, Shao H, Mazumdar M, Greenfield JP, Weinstein S, et al: Vagus nerve stimulation vs. corpus callosotomy in the treatment of Lennox-Gastaut syndrome: a meta-analysis. Seizure 22:3-8, 2013

8. Nordgren RE, Reeves AG, Viguera AC, Roberts DW: Corpus callosotomy for intractable seizures in the pediatric age group. Arch Neurol 48:364-372, 1991

9. Reutens DC, Bye AM, Hopkins IJ, Danks A, Somerville E, Walsh J, et al: Corpus callosotomy for intractable epilepsy: seizure outcome and prognostic factors. Epilepsia 34:904909, 1993

10. Roberts DW: The role of callosal section in surgical treatment of epilepsies. Neurosurg Clin N Am 4:293-300, 1993

11. Rosenfeld WE, Roberts DW: Tonic and atonic seizures: what's next-VNS or callosotomy? Epilepsia 50 (Suppl 8):25-30, 2009

12. Shim KW, Lee YM, Kim HD, Lee JS, Choi JU, Kim DS: Changing the paradigm of 1-stage total callosotomy for the treatment of pediatric generalized epilepsy. J Neurosurg Pediatr 2:29-36, 2008

13. Spencer SS, Spencer DD, Sass K, Westerveld M, Katz A, Mattson R: Anterior, total, and two-stage corpus callosum section: differential and incremental seizure responses. Epilepsia 34:561-567, 1993

14. Wilson DH, Reeves AG, Gazzaniga MS: "Central" commissurotomy for intractable generalized epilepsy: series two. Neurology 32:687-697, 1982

15. Yaşargil MG, Türe U, Yaşargil DC: Surgical anatomy of supratentorial midline lesions. Neurosurg Focus 18(6B):E1, 2005

\section{Author Contributions}

Conception and design: Ito. Acquisition of data: Niimura, Takamizawa, Shimizu. Analysis and interpretation of data: Ito. Drafting the article: Ito. Administrative/technical/material support: Morino. Study supervision: Morino.

\section{Correspondence}

Hirotaka Ito, Department of Neurosurgery, Tokyo Metropolitan Neurological Hospital, Musashidai 2-6-1, Fuchu-shi, Tokyo, Japan. email: xb9r715@hotmail.com. 